\title{
Patterns of care study of brachytherapy in New South Wales: malignancies of the uterine corpus
}

\author{
Stephen R. Thompson, MBBS, PhD, FRANZCRI,2,3, Prof. Geoff P. Delaney, MBBS, MD, PhD, FRANZCR!1,3, \\ Gabriel S. Gabriel, MBBS, MPH, FAFPHM ${ }^{1,3}$, Prof. Michael B. Barton, OAM, MBBS, MD, FRANZCR,3 \\ 'Collaboration for Cancer Outcomes Research and Evaluation (CCORE), Liverpool Hospital, ${ }^{2}$ Department of Radiation Oncology, Prince of \\ Wales Hospital, ${ }^{3}$ University of New South Wales, ${ }^{4}$ University of Western Sydney, Sydney, NSW, Australia
}

\begin{abstract}
Purpose: There are limited data at a population level on adjuvant brachytherapy (BT) practice for uterine corpus malignancies. The aims of the current study were to describe BT practice for this disease in New South Wales (NSW), to assess quality of BT, and to determine if a caseload effect on quality exists.

Material and methods: Patient, tumour, and treatment related data were collected from all nine NSW radiation oncology departments that treated patients with BT. Included patients had malignancy of the uterine corpus with treatment including BT. Brachytherapy quality was assessed using published quality benchmarks. Higher gynaecological BT caseload departments were compared with lower caseload departments.

Results: One hundred sixty-three NSW residents with gynaecological cancer were treated with BT. The four higher gynaecological BT caseload departments treated a median of 25.5 NSW residents (range 22-38), compared to median 10 (range 3-18) in the five lower caseload departments. Seventy-five patients underwent BT for uterine malignancies. Most patients had early stage endometrioid disease and were treated adjuvantly with intravaginal cylinders using high-dose-rate BT. Doses were in accordance with guideline recommendations in $83 \%$ of cases, and BT was appropriately indicated in $76 \%$ of cases. Higher caseload departments were more likely to treat with guideline doses (96\% vs. $53 \%, p<0.001)$ but there was no difference in compliance rate with treatment indications $(p=0.75)$.

Conclusions: Brachytherapy patients, techniques, and numbers/unit for uterine corpus malignancies were similar in NSW compared to the USA but more dispersed than in Western Europe. Doses prescribed may not be optimal in lower gynaecological BT caseload departments - the significance of this represents an area in which more research is needed.
\end{abstract}

J Contemp Brachytherapy 2015; 7, 3: 224-230 DOI: $10.5114 /$ jcb.2015.52623

Key words: brachytherapy, caseload volume, endometrial cancer, quality, patterns of care studies.

\section{Purpose}

Retrospective Patterns of Care Studies (POCS) seek to improve the quality of technical care by examining processes, structures and/or outcomes of care. The rationale is that differences exist in care; that these differences can be measured against explicit criteria and against each other; that deficiencies in care may affect outcome, and that these deficiencies, having been identified, are correctable, thereby improving patient outcome [1]. We performed the first comprehensive, population-based brachytherapy (BT) POCS in Australia. Collection of these data enabled an overview of actual New South Wales (NSW) BT use for comparison with our model of optimal BT utilization, showing that gynaecological BT is underutilized in NSW, reported previously [2]. The other aim of this POCS was to study patterns of practice of BT in NSW, with a particular focus on quality of care.
Herein, we focus on these results as they pertain to BT for uterine corpus malignancies.

Worldwide, gynaecological cancer in total is responsible for over $14 \%$ of female cancer deaths, more than that of breast cancer or female lung cancer. The international incidence of endometrial cancer in 2008 totalled 287,000 , making it the $6^{\text {th }}$ commonest female malignancy [3]. Brachytherapy is an integral part of endometrial cancer treatment $[4,5]$, and the publication of the results of the PORTEC2 randomized trial, showing equivalence between adjuvant BT compared to pelvic external beam radiotherapy (EBRT) for intermediate risk endometrial cancer, is likely to further increase the utilization and importance of BT for this disease [6]. Despite this, current BT practice for uterine corpus malignancies is not well studied at a population level. There have been no POCS addressing endometrial cancer in Australia, and only
Address for correspondence: Stephen R. Thompson, MBBS, PhD, FRANZCR, Department of Radiation Oncology, Prince of Wales Hospital, High St, Randwick, NSW 2031, Australia, phone: +61 29382 2501, fax: +61 29382 2550, ఐ e-mail: stephen.thompson@sesiahs.health.nsw.gov.au
Received: 12.11 .2014

Accepted: 03.06.2015

Published: 25.06.2015 
one survey of Australian gynaecological BT protocols, which provided limited data on how these patients are being treated with BT [7]. Similarly, there is very limited international literature detailing patterns of radiotherapy practice for uterine corpus malignancies [8-11].

The current study therefore had three aims, which were to: 1) describe the Patterns of Care of BT for malignancies of the uterine corpus in NSW; 2) assess the quality of BT in NSW for uterine corpus malignancies; and 3) determine whether there is a caseload effect on quality of BT for uterine corpus malignancies.

\section{Material and methods}

In 2005, a retrospective BT POCS was performed. The population for this manuscript was all NSW residents with a diagnosis of malignancy of the uterine corpus, treated with BT in NSW in 2003. Patients with cancer of the uterine cervix were excluded. New South Wales is the largest state of Australia, with a population of 6.6 million in 2003, 63\% of whom lived in Sydney [12]. Site visits and data collection by the principal investigator were made to all nine radiation oncology departments in NSW that deliver BT. Collected data were entered into an Access database and then transferred into SPSS v15.0. Logic checks and descriptive analyses were performed to identify data errors and inconsistencies.

Quality of BT treatment was assessed using the published benchmarks, assessing concordance with NSW recommended doses and with treatment indications [4]. Adjuvant monotherapy doses recommended by the NSW guidelines were for low-dose-rate (LDR) BT 50-60 Gy, or for high-dose-rate (HDR) BT 30-40 Gy in 4-6 fractions. Doses were compared using the linear quadratic equation to convert HDR BT doses to equivalent LDR doses, which are approximately equivalent to standard fractionation at 2 Gy per fraction (EQD). For late effects, $\alpha / \beta=3$, and for early effects and modelling of tumour control $\alpha / \beta=$ $=10$ [13]. The HDR monotherapy doses recommended are thus EQD2 $(\alpha / \beta=10)=44-56 \mathrm{~Gy}$. Combined modal- ity treatment doses recommended were 45-54 Gy EBRT in 1.8-2.0 Gy fractions with BT boost by LDR $20 \mathrm{~Gy}$, or by HDR 15-18 Gy/2-4 fractions, EQD2 $(\alpha / \beta=10)=67-76$ Gy. Prescribed doses were scored as appropriate if they fell within the range of these recommendations. Note that the NSW recommendations are similar to those of the American Brachytherapy Society but with a broader range [5]. Treatment with adjuvant BT was scored as potentially inappropriate if delivered to patients with stage I disease without adverse pathological features (stage IC, lymphovascular space invasion, grade 3 , or close margins) giving an indication for adjuvant RT (BT with or without EBRT) [4]. Brachytherapy dose and treatment indication appropriateness was compared between higher and lower gynaecological BT caseload departments, defined as the four departments that performed more gynaecological BT compared to the five departments that performed fewer.

Categorical variables were compared using the chisquare test. The study was approved by all relevant Human Research Ethics Committees and carried out in accordance with the Helsinki Declaration. Patient and departmental data were de-identified.

\section{Results}

In NSW in 2003, there were 15 radiation oncology departments, of which $9(60 \%)$ treated patients with BT, all administering gynaecological BT. After exclusion of 20 patients resident interstate, 163 NSW gynaecological cancer patients were treated with BT in 2003 (Table 1), most for cervical cancer $(n=76)$ or uterine malignancies $(n=75)$. The four higher gynaecological BT caseload departments treated $111(68 \%)$ of the patients, median 25.5 per department (range 22-38). The five lower gynaecological BT caseload departments treated the remaining 52 (32\%) patients, median 10 each (range 3-18). Mean age of patients with uterine malignancies was 65 (range 42-88) years. Patient stage and histology are shown in Table 2: $77 \%$ had early stage disease and $80 \%$ had non-papillary serous/clear cell adenocarcinomas.

Table 1. Gynaecological brachytherapy activity, New South Wales cancer patients, 2003

Institution Gynaecological primary tumour site

Total

\begin{tabular}{|c|c|c|c|c|c|c|}
\hline & Vagina & Cervix & Uterus & Ovary & $n$ & (\%) \\
\hline 1 & 2 & 12 & 7 & 1 & 22 & 13 \\
\hline 2 & - & 3 & - & - & 3 & 2 \\
\hline 3 & 2 & 14 & 21 & 1 & 38 & 23 \\
\hline 4 & - & 4 & 1 & - & 5 & 3 \\
\hline 5 & 1 & 8 & 9 & - & 18 & 11 \\
\hline 6 & 2 & 8 & 6 & - & 16 & 10 \\
\hline 7 & 1 & 15 & 10 & - & 26 & 16 \\
\hline 8 & 1 & 1 & 8 & - & 10 & 6 \\
\hline 9 & - & 11 & 13 & 1 & 25 & 15 \\
\hline Total & 9 & 76 & 75 & 3 & 163 & 100 \\
\hline
\end{tabular}


Table 2. New South Wales uterine corpus brachytherapy patients. Tumour stage and histology

\begin{tabular}{cccccc}
$\begin{array}{c}\text { FIGO } 2000 \\
\text { Stage }\end{array}$ & Adenocarcinoma, non-PS/CC & Adenocarcinoma, PS/CC & MMMT & Sarcoma & Total \\
\hline IA & 3 & - & - & - & $3(4 \%)$ \\
\hline IB & 17 & 2 & 1 & - & $20(27 \%)$ \\
\hline IC & 14 & 1 & - & - & $9(21 \%)$ \\
\hline IIA & 6 & 3 & - & - & $10(13 \%)$ \\
\hline IIB & 9 & 1 & - & $8(11 \%)$ \\
\hline IIIA & 6 & - & - & - \\
\hline IIIB & - & - & - & - & $3(4 \%)$ \\
\hline IIIC & 2 & - & - & - & - \\
\hline IVA & - & - & - & 1 & $1(1 \%)$ \\
\hline IVB & - & - & 1 & 1 & $5(7 \%)$ \\
\hline n/av & 3 & $7(9 \%)$ & $6(8 \%)$ & $2(3 \%)$ & $75(100 \%)$
\end{tabular}

FIGO - Federation Internationale de Gynecologie et d'Obstetrique, PS/CC - papillary serous or clear cell carcinoma, MMMT - malignant mixed mullerian tumour; n/av-not available

As shown in Table 2, five patients had recurrent uterine corpus malignancy treated with BT. Two were treated palliatively, and three underwent definitive combination EBRT and BT. Seventy patients had curative treatment, including BT for their initial disease presentation and are the subject of the remainder of the report in this section. Sixty-nine of these patients had adjuvant treatment post hysterectomy and one patient underwent neo-adjuvant BT.

Intra-vaginal cylinders with library plans were used by six department to treat $64(91 \%)$ of the 70 patients. One department used ovoids, treating $5(7 \%)$ patients, and one department treated one patient only with tandem and ovoids neoadjuvantly. These two departments used orthogonal film based planning. Low-dose-rate caesium BT was used by two departments to treat 13 (19\%) of the patients, pulsed-dose-rate (PDR) iridium was used by one department (treating as LDR) to treat $1(1 \%)$ of the patients, and HDR iridium BT was used by five departments treating $56(80 \%)$ of the patients.

Twelve patients were treated with adjuvant LDR BT monotherapy at two departments. All were dosed to the mucosal surface, and 10 of the 12 patients were dosed to 60 Gy (range 21-60 Gy). Adjuvant HDR BT monotherapy was used to treat 44 patients at five other departments. Four of these patients had dose prescribed to $0.5 \mathrm{~cm}$ below the vaginal mucosal surface: 15 Gy in $3 \#$ or 30 Gy in $6 \#$. The other 40 patients were dosed to the mucosal surface (see Table 3), with median EQD2 being 44 Gy (range 24-67 Gy).

Thirteen of the 70 (19\%) patients underwent adjuvant pelvic EBRT (in addition to BT) at five of the nine departments (Table 4). Median total EQD2 at the vaginal mucosal surface was 74 Gy (range 61-91 Gy).

As measured by conformity to guideline recommendations, doses were appropriate in $83 \%$ of patients

Table 3. Adjuvant high-dose-rate brachytherapy monotherapy dosing schedules

\begin{tabular}{|c|c|c|c|c|c|c|c|}
\hline \multirow{2}{*}{$\begin{array}{c}\text { Dose (Gy) } \\
\text { and fractions }\end{array}$} & \multirow[t]{2}{*}{ EQD2 (Gy) } & \multicolumn{5}{|c|}{ Number of patients } & \multirow[t]{2}{*}{ Tota } \\
\hline & & Dept 3 & Dept 5 & Dept 6 & Dept 8 & Dept 9 & \\
\hline $18 / 3$ & 24 & - & - & 2 & - & - & 2 \\
\hline $28 / 4$ & 40 & - & - & - & 1 & - & 1 \\
\hline $30 / 4$ & $44^{\dagger+}$ & 21 & - & - & 3 & - & 24 \\
\hline $34 / 4$ & $53^{\dagger+}$ & - & 5 & - & 1 & 5 & 11 \\
\hline $36 / 6$ & $48^{t+}$ & - & - & 1 & - & - & 1 \\
\hline $43 / 5$ & 67 & - & - & - & - & 1 & 1 \\
\hline
\end{tabular}

Gy-Gray, EQD2 - Equivalent dose at $2 \mathrm{~Gy} / \#, \alpha / \beta=10$ - approximately equivalent to LDR total dose [14]; ${ }^{+}$- brachytherapy doses were prescribed to vaginal mucosal surface; ${ }^{+t}$ - brachytherapy dose within guideline recommendations [5], Dept-Department 
Table 4. Combination external beam radiotherapy and brachytherapy adjuvant dosing schedules

\begin{tabular}{|c|c|c|c|c|c|}
\hline Department & $\begin{array}{l}\text { Pelvic EBRT dose } \\
\text { fractions (Gy/\#) }\end{array}$ & $\begin{array}{l}\text { Brachytherapy dose } \\
\text { rate }\end{array}$ & $\begin{array}{c}\text { Brachytherapy } \\
\text { dose and fractions } \\
(\mathrm{Gy} / \#)^{\dagger}\end{array}$ & $\begin{array}{c}\text { Total EQD2 at } \\
\text { vaginal mucosal } \\
\text { surface } \\
\end{array}$ & Number of patients \\
\hline \multirow[t]{2}{*}{5} & $45 / 25$ & $\mathrm{HDR}$ & $12 / 2$ & 61 & 2 \\
\hline & $50.4 / 28$ & & & 66 & 1 \\
\hline 6 & $50.4 / 28$ & HDR & $26 / 3$ & 91 & 1 \\
\hline 7 & $50.4 / 28$ & LDR & $20 / 1$ & $70^{\dagger+}$ & 1 \\
\hline 8 & $?$ & HDR & $24 / 4$ & $?$ & 1 \\
\hline 9 & $50.4 / 28$ & HDR & $18 / 3$ & $74^{\dagger+}$ & 7 \\
\hline
\end{tabular}

EBRT - external beam radiotherapy, Gy - Gray, EQD2 - equivalent dose at $2 \mathrm{~Gy} / \#, \alpha / \beta=10[14],{ }^{\dagger}$ - brachytherapy doses are normalised to vaginal mucosal surface, ${ }^{+t}$ - total dose within guideline recommendations [5]

(Fig. 1). Higher gynaecological BT caseload departments were more likely to treat to an appropriate dose (45 of 47 patients, 96\%) than lower BT caseload departments (10 of 19 patients, $53 \%), p<0.001$. There were no significant differences by BT dose rate $(p=0.33)$.

Treatment with adjuvant BT was scored as potentially inappropriate if delivered to patients with stage I disease without adverse pathological features (stage IC, lymphovascular invasion, grade 3 , or close margins) giving an indication for adjuvant RT (BT with or without EBRT) (5): 8 of 34 cases $(24 \%)$, with no significant difference by gynaecological BT caseload $(p=0.75)$ - Fig. 2 .

\section{Discussion}

The patients in this study were a population-based sample, and were similar to those in the SEER database, with $80 \%$ having adenocarcinomas of non-papillary serous/clear cell subtype, and $82 \%$ of these being stage I-II; compared to SEER: $90 \%$ of uterine malignancies being these non-high risk histological subtypes and $84 \%$ of

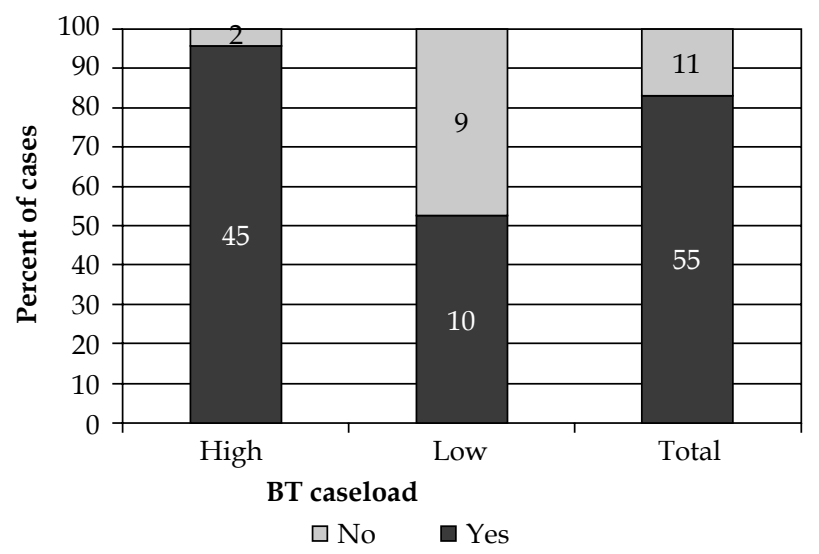

Fig. 1. Appropriateness of adjuvant radiotherapy dose, by departmental gynaecological brachytherapy caseload volume $(p<0.001)$. Appropriate dose was as recommended by New South Wales guidelines [5] these stage I-II [14]. Treatment in NSW was also similarly distributed to that in the USA, with $91 \%$ of NSW patients undergoing adjuvant BT being treated with an intra-vaginal cylinder, compared to the American Brachytherapy Society survey of adjuvant vaginal BT for endometrial cancer, also covering 2003 [8]: vaginal cylinders were used by $75 \%$ of respondents. In the two areas, a similarly low proportion of departments used LDR: 2 of 8 (25\%) NSW departments compared with $31 \%$ of respondents in the ABS survey. LDR and HDR appear to be equally effective and safe, but the latter has well-recognized advantages in terms of patient convenience and comfort [15]. In our POCS, there was no difference between HDR and LDR prescriptions in terms of proportions of patients being treated to guideline recommended doses. There was, however, a large difference in the assessment of normal tissue doses: in the NSW POCS only the two departments using LDR BT, and ovoids recorded bladder and rectal doses in 8 of the $70(11 \%)$ patients, compared to $78 \%$ of respondents for bladder and $80 \%$ for rectal doses in the ABS survey. The difference may be attributable to differences

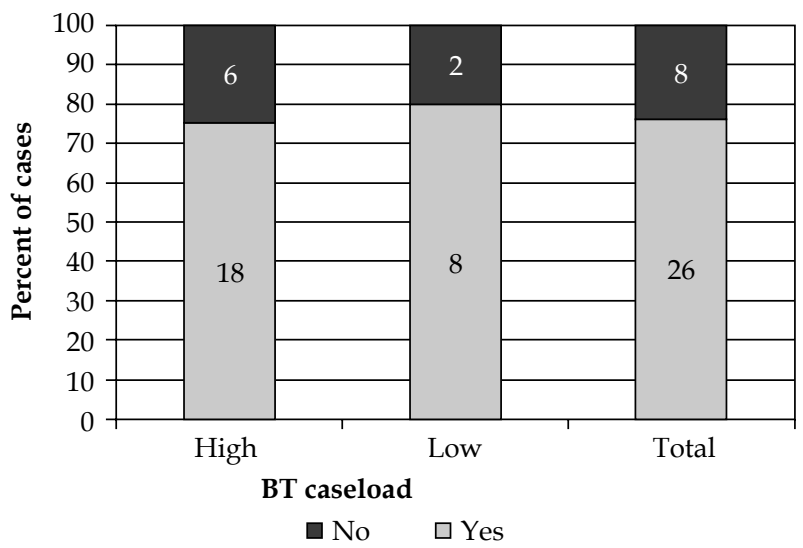

Fig. 2. Appropriateness of adjuvant brachytherapy indication, by departmental gynaecological brachytherapy caseload volume - stage I endometrioid adenocarcinoma of the uterus $(p=0.75)$. Appropriateness of brachytherapy indication was as recommended by New South Wales guidelines [5] 
in guideline recommendations; the NSW guidelines make no recommendations regarding dose to normal tissue reporting [4]. The ABS does recommend doses to organ at risk reporting, although the ABS document does hedge the recommendation with the comment "...with the use of fixed geometry applicators, such as vaginal cylinders, localization radiographs need not be performed" [5]. Other reasons for the lack of normal tissue dose reporting in the HDR treated NSW POCS may be the reported low rate of rectal and urinary complications experienced by this group of patients [15].

There was a wide variation in adjuvant doses delivered in NSW, with 15 different total doses prescribed across the eight treating departments, with the EQD2 dose $(\alpha / \beta=10)$ ranging from $21 \mathrm{~Gy}$ to $91 \mathrm{~Gy}$. This variation in dose may reflect the lack of published dose response data, but it is concerning that in most departments more than one dose had been prescribed. There may have been clinical reasons for individualization of doses in particular patients. The ABS survey also revealed a wide variation in dose prescriptions and fractionations [8]. Overall, when measured against the recommendations of the NSW guidelines [4], dose prescriptions were within the recommended range in $83 \%$ of patients. Higher gynaecological BT caseload departments were significantly more likely to treat to a recommended dose than lower caseload departments ( $96 \%$ vs. $53 \%$ of cases, $p<0.001)$. It is recognized that the NSW POCS assessed treatment for 2003 but the NSW guidelines were not published until 2004. Nevertheless, these guidelines were widely distributed in NSW in various drafts for some ten years prior to publication and provide a reasonable summation of what was considered benchmark treatment for gynaecological malignancies in NSW in the early 2000s. A further explanation may be that the NSW guidelines simply reflect the practice of the larger gynaecological departments, and hence the greater likelihood of the higher gynaecological BT caseload departments treating according to these guidelines. When assessing the appropriateness of adjuvant $B T$ indication for stage I patients in comparison to the NSW guidelines, there was no significant difference between departments by caseload, with treatment being appropriate in $76 \%$ of cases overall (Fig. 2). This is higher than that determined by applying the same criteria to a recent SEER study, in which the use of adjuvant BT (with or without EBRT) was appropriate in only $60 \%$ of treated stage I patients. Although, some of the patients potentially inappropriately treated in that study may have had other relative indications not recorded in the manuscript, such as lymphovascular invasion [10].

Whilst there are data correlating outcome with caseload in treating cervical cancer [16-18], and there have been two reports showing potential benefits from sub-specialty surgical training on outcome in uterine malignancies $[19,20]$, this is the first report assessing the effect of radiotherapy provider caseload on treatment for this disease. Gynaecological BT, specifically BT for uterine malignancies, is generally more dispersed in NSW and the USA compared to Western Europe, with fewer cases treated per centre (Table 5). Although this is potentially a factor that may adversely impact on the quality of cervical BT and outcomes [16-18], it may not be an important issue in the treatment quality of uterine cancers. A further

Table 5. New South Wales gynaecological brachytherapy caseload per centre and comparison with other jurisdictions

\begin{tabular}{|c|c|c|c|c|c|c|c|c|}
\hline \multirow[t]{2}{*}{$\begin{array}{l}\text { Tumour } \\
\text { site }\end{array}$} & \multirow{2}{*}{$\begin{array}{c}\text { Cases/ } \\
\text { Centre/ } \\
\text { Year }\end{array}$} & \multicolumn{3}{|c|}{$\begin{array}{l}\text { NSW } \\
2003\end{array}$} & \multirow[t]{2}{*}{$\begin{array}{c}\text { ABS, } 1992 \\
{[21]}\end{array}$} & \multirow[t]{2}{*}{$\begin{array}{c}\text { UK, } 2004 \\
{[22]}\end{array}$} & \multirow[t]{2}{*}{$\begin{array}{l}\text { Italy, } 2002 \\
{[23]}\end{array}$} & \multirow[t]{2}{*}{$\begin{array}{c}\text { Spain, } 2002 \\
\text { [23] }\end{array}$} \\
\hline & & All & $\begin{array}{c}\text { Higher } \\
\text { caseload }\end{array}$ & $\begin{array}{c}\text { Lower } \\
\text { caseload }\end{array}$ & & & & \\
\hline \multirow[t]{4}{*}{ All } & Mean & 18 & 27.8 & 10.4 & - & 61 & 35 & 76 \\
\hline & Median & 18 & 25.5 & 10 & - & - & - & - \\
\hline & Range & $3-38$ & $22-38$ & $3-18$ & - & $13-174$ & $\begin{array}{c}21-48 \\
95 \% \mathrm{Cl}\end{array}$ & $\begin{array}{c}54-99 \\
95 \% \mathrm{Cl}\end{array}$ \\
\hline & $>25$ cases & $22 \%$ & $50 \%$ & $0 \%$ & - & $88 \%$ & - & - \\
\hline \multirow{4}{*}{$\begin{array}{l}\text { Uterine } \\
\text { cervix }\end{array}$} & Mean & 8.4 & 13.0 & 4.8 & - & - & - & - \\
\hline & Median & 8 & 13 & 4 & - & 19 & - & - \\
\hline & Range & $1-15$ & $11-15$ & $1-8$ & - & $9-45$ & - & - \\
\hline & $>10$ cases & $44 \%$ & $100 \%$ & $0 \%$ & $37 \%$ & - & - & - \\
\hline \multirow{4}{*}{$\begin{array}{l}\text { Uterine } \\
\text { corpus }\end{array}$} & Mean & 9.4 & 12.8 & 4.8 & - & - & - & - \\
\hline & Median & 8.5 & 11.5 & 6 & - & - & - & - \\
\hline & Range & $0-21$ & $7-21$ & $0-9$ & - & - & - & - \\
\hline & $>10$ cases & $25 \%$ & $50 \%$ & $0 \%$ & $28 \%$ & - & - & - \\
\hline
\end{tabular}

NSW - New South Wales, ABS - American Brachytherapy Society, UK - United Kingdom, 95\% CI - 95\% confidence interval, “-” - not available 
consideration is that actual gynaecological BT utilization is close to our previously modelled optimal rate in the UK and Spain (where gynaecological BT is concentrated), but sub-optimal in Italy (also concentrated) and in NSW and SEER (dispersed). The significance of concentration or dispersal of uterine BT per centre and whether this may impact on utilization of BT is unknown [2].

It should be noted that almost all patients in this study underwent adjuvant vaginal vault BT, with only three patients having definitive treatment. Definitive RT with BT for endometrial cancer is used rarely, but can often be curative [24]. This study did have a number of limitations. It was retrospective, involved small patient numbers, represents state-of-play in 2003, and therefore not necessarily reflecting current NSW practice. However, although there have been dramatic changes in cervical brachytherapy with widespread incorporation of MRI assisted 3D planning, we contend that there have been minimal changes in vaginal vault brachytherapy for endometrial cancer. This is likely secondary to the good results achieved with vault brachytherapy for this disease in terms of high rates of local control and minimal radiation related morbidity. Nevertheless, there is a paucity of Patterns of Care data, either of current or of recent practise, to confirm this. Robust vaginal vault BT treatment quality indicators against which performance can be measured are lacking. Importantly, this study reports technical factors but not outcome data. These are areas in which future research is likely to be beneficial.

The study has a number of strengths. Although numerous studies have found a correlation between provider caseload for prostate and cervical BT quality and/ or outcomes, none have assessed whether this caseload effect exists for BT in the context of uterine corpus malignancies. This is the first paper to do so. It adds to the sparse population level data on brachytherapy use in this disease. It is the final in a series of three publications summarising brachytherapy Patterns of Care in New South Wales, and completes the picture with the other two publications also assessing patterns of care for brachytherapy and caseload effects on quality for cervical [18] and prostate cancer [25].

\section{Conclusions}

In the case of BT for uterine corpus malignancies, tumour types and BT techniques in NSW in 2003 were similar to those in the USA. A wide range of adjuvant vaginal vault doses were delivered, although usually within the recommended range. New South Wales gynaecological BT (like that of the USA but not Italy, Spain or the UK) was dispersed amongst a large number of treating departments, although the significance of this is not clear: higher gynaecological caseload departments were more likely than lower caseload departments to treat to recommended doses (in the absence of published dose response data), but were equally likely to treat patients with guideline recommended indications. Further research as to treatment quality indicators for BT in this disease is needed.

\section{Acknowledgments}

The authors thank the Directors of Radiation Oncology Departments in New South Wales who provided access to patient data.

\section{Meeting Presentations}

This manuscript is based on data presented at the following Scientific Meetings: 1. The Royal Australian and New Zealand College of Radiologists $56^{\text {th }}$ Annual Scientific Meeting, Sydney, October 2005, and 2. The Royal Australian and New Zealand College of Radiologists $61^{\text {st }}$ Annual Scientific Meeting, Perth, October 2010 [26].

\section{Disclosure}

Authors report no conflict of interest.

\section{References}

1. Coia L, Hanks G. Quality assessment in the USA: how the Patterns of Care Study has made a difference. Sem Rad Oncol 1997; 7: 146-156.

2. Thompson SR, Delaney GP, Gabriel GS et al. Estimation of the optimal brachytherapy utilization rate in the treatment of gynaecological cancers and comparison with patterns of care. Int J Radiat Oncol Biol Phys 2013; 85: 400-405.

3. Ferlay J, Shin HR, Bray F et al. Estimates of worldwide burden of cancer in 2008: GLOBOCAN 2008. Int J Cancer 2010; 127: 2893-2917.

4. NSW Gynaecological Oncology Study Group. Gynaecological Oncology Clinical Practice Guidelines. 2004. Sydney, NSW Gynaecological Oncology Study Group. Ref Type: Serial (Book, Monograph).

5. Nag S, Erickson B, Parikh S et al. The American Brachytherapy Society recommendations for high-dose-rate brachytherapy for carcinoma of the endometrium. Int J Radiat Oncol Biol Phys 2000; 48: 779-790.

6. Nout RA, Smit VTHBN, Putter H et al. Vaginal brachytherapy versus pelvic external beam radiotherapy for patients with endometrial cancer of high-intermediate risk (PORTEC-2): an open-label, non-inferiority, randomised trial. Lancet 2010; 375: 816-823.

7. MacLeod C, Cheuk R, Dally M et al. Australian high-doserate brachytherapy protocols for gynaecological malignancy. Australas Radiol 2001; 45: 43-48.

8. Small W, Erickson B, Kwakwa F. American Brachytherapy Society survey regarding practice patterns of postoperative irradiation for endometrial cancer: current status of vaginal brachytherapy. Int J Radiat Oncol Biol Phys 2005; 63: 1502-1507.

9. Kwon JS, Carey MS, Cook EF et al. Patterns of practice and outcomes in intermediate- and high-risk stage I and II endometrial cancer: a population-based study. Int J Gynecol Cancer 2007; 17: 433-440.

10. Lee C, Szabo A, Shrieve D et al. Descriptive nomograms of adjuvant radiotherapy use and patterns of care analysis for stage I and II endometrial adenocarcinoma: A surveillance, epidemiology, and end results population study. Cancer 2007; 110: 2092-2100.

11. Small W, Du Bois A, Bhatnagar S et al. Practice patterns of radiotherapy in endometrial cancer among member groups of the gynecologic cancer intergroup. Int J Gynecol Cancer 2009; 19: 395-399.

12. Australian Bureau of Statistics. Australian Bureau of Statistics National Regional Profile. http://www.abs.gov.au/. 2005. 24-11-2005. Ref Type: Electronic Citation. 
13. Lang S, Kirisits C, Dimopoulos J et al. Treatment planning for MRI assisted brachytherapy of gynecologic malignancies based on total dose constraints. Int J Radiat Oncol Biol Phys 2007; 69: 619-627.

14. National Cancer Institute (Cancer Statistics Branch). SEER ${ }^{*}$ Stat 5.2.2. Surveillance, Epidemiology and End Results Cancer Incidence Public-Use Database, 1973-2001. 2004. Bethesda, US Department of Health and Human Services. Ref Type: Report.

15. MacLeod C, Fowler A, Duval P et al. High-dose-rate brachytherapy alone post-hysterectomy for endometrial cancer. Int J Radiat Oncol Biol Phys 1998; 42: 1033-1039.

16. Eifel PJ, Moughan J, Erickson B et al. Patterns of radiotherapy practice for patients with carcinoma of the uterine cervix: a patterns of care study. Int J Radiat Oncol Biol Phys 2004; 60: 1144-1153.

17. Inoue T. Quality assurance of radiotherapy and its clinical assessment. Japan J Clin Oncol 2002; 32: 497-505.

18. Thompson SR, Delaney GP, Gabriel GS et al. Patterns of care study of brachytherapy in New South Wales: cervical cancer treatment quality depends on caseload. J Contemp Brachytherapy 2014; 6: 28-32.

19. Roland PY, Kelly FJ, Kulwicki CY et al. The benefits of a gynecologic oncologist: a pattern of care study for endometrial cancer treatment. Gynecol Oncol 2004; 93: 125-130.

20. Macdonald OK, Sause WT, Jeffery Lee R et al. Does oncologic specialization influence outcomes following surgery in early stage adenocarcinoma of the endometrium? Gynecol Oncol 2005; 99: 730-735.

21. Nag S, Owen JB, Farnan N et al. Survey of brachytherapy practice in the United States: A report of the clinical research committee of the American Endocurietherapy Society. Int J Radiat Oncol Biol Phys 1995; 31: 103-107.

22. Board of the Faculty of Clinical Oncology, The Royal College of Radiologists. The Role and Development of Brachytherapy Services in United Kingdom. http://www.rcr.ac.uk. 2007. Ref Type: Electronic Citation.

23. Guedea F, Ventura M, Marchetti C et al. Patterns of care for brachytherapy in Europe, in Spain and Italy: comparative results. Tumori 2007; 93: 439-444.

24. Van der Steen-Banasik E. Primary brachytherapy as a radical treatment for endometrial carcinoma. J Contemp Brachytherapy 2014; 6: 106-112.

25. Thompson SR, Delaney GP, Gabriel GS et al. Prostate brachytherapy in New South Wales: patterns of care study and impact of caseload on treatment quality. J Contemp Brachytherapy 2014; 6: 344-349.

26. Thompson S, Delaney G, Gabriel G et al. NSW brachytherapy patterns of care study: Impact of caseload on treatment quality. J Med Imag Radiat Oncol 2010; 54: A82. 